\section{NEUROSCIENCE}

\section{Pay attention to the neurons}

Many animals, including humans, can control the firing of certain neurons with conditioning, and researchers now show that this can lead to enhanced attention.

Robert Schafer and Tirin Moore at Stanford University in California trained two monkeys to increase and decrease the firing of neurons in an area of the brain known to be involved in eye movement, the frontal eye field. They then measured the activity at 94 sites in this region while the monkeys performed a visual search task. During the trials in which the monkeys had to increase their neuronal firing rate, their search performance improved in the part of the visual field represented by the controlled part of the frontal eye field, indicating greater attention.

The authors suggest that this may explain why neurofeedback, in which patients are trained to control their neurophysiology, might be able to treat attention deficit disorder.

Science doi:10.1126/ science.1199892 (2011)

\section{STEM CELLS}

\section{Gene repair tool} for stem cells

By correcting disease-causing mutations in stem cells from patients with certain degenerative disorders, researchers could generate new disease models and treatments. Juan Carlos Izpisúa Belmonte at the Salk Institute for Biological Studies in La Jolla, California, and his colleagues engineered an adenovirus that efficiently fixes mutated portions of

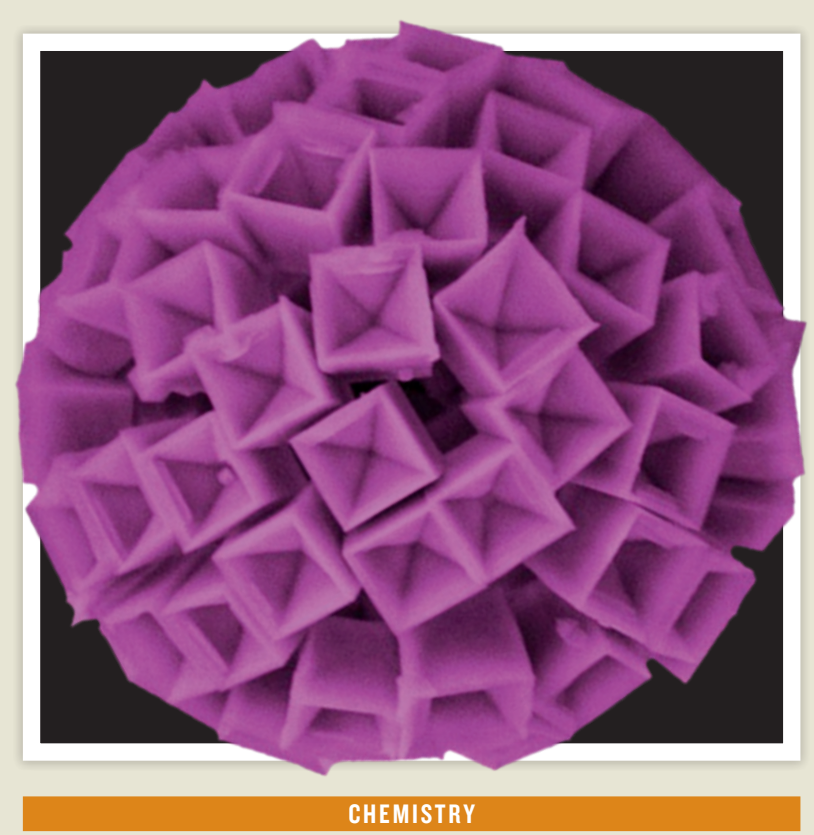

\title{
Crystals on water's edge
}

Certain salt crystals are normally cube-shaped, but can take on a new shape and grow on the surface of tiny water droplets.

Zhongping Zhang, Suhua Wang and their colleagues at the Chinese Academy of Sciences in Hefei mixed a water-based solution of either sodium chloride or potassium chloride with cyclohexane and acetone. After shaking, hollow spheres formed that were made up of individual 'hopper-like' crystals of the salt (pictured). The spheres probably formed at the interface between the organic solvents and the water droplets that contained the salt.

The technique may produce new crystal structures for other water-soluble compounds, and could aid in understanding crystal growth mechanisms, the authors suggest.

Angew. Chem. Int. Edn doi:10.1002/anie.201101704 (2011)

the lamin A ( LMNA) gene in stem cells created by reprogramming patients' cells. Corrected cells from patients with HutchinsonGilford progeria syndrome, an accelerated ageing disorder, produced normal connective tissue cells.

The same virus also repaired different $L M N A$ mutations underlying a milder premature-ageing disease, atypical Werner syndrome. Moreover, it mended
LMNA mutations in adult mesenchymal stem cells, a focus of current regenerative therapies.

Cell Stem Cell doi:10.1016/

j.stem.2011.04.019 (2011) ECOLOGY

\section{Speciation drives plant extinction}

An animal's risk of extinction is associated with species traits such as body size and geographic range - but this is not the case for plants. Jonathan Davies at McGill University in Montreal, Canada, and his colleagues report that, contrary to patterns seen in animals, threatened plants tend to be young species in rapidly evolving lineages.

The team studied the evolutionary history of threatened plant species on the Cape of South Africa - a plant biodiversity hot spot. Anthropogenic drivers, such as habitat loss, do not seem to endanger plants as they do animals. The authors suggest that conservation strategies for animals and plants should use different assessment criteria.

PloS Biol. 9, e1000620 (2011)

\section{MOLECULAR NEUROSCIENCE}

\section{Bitter breath for lung airways}

Lining the lower airways are 'brush' cells that can sense bitter molecules and send signals to regulate breathing, perhaps providing a defence against bacteria, which produce bitter compounds.

Gabriela Krasteva at Justus Liebig University in Giessen, Germany, and her colleagues found that brush cells in the tracheae of mice express protein receptors and other key molecules involved in bitter-taste signalling. The cells make contact with vagal nerve fibres, which connect key visceral organs to the brain, and send signals to the nerves by releasing the neurotransmitter acetylcholine. When the researchers administered a bitter substance directly to the airway surfaces of mice, the animals' respiratory rate dropped suddenly.

Proc. Natl Acad. Sci. USA doi:10.1073/pnas.1019418108 (2011) 